\title{
Sociología de la Infancia: las niñas y los niños como actores sociales ${ }^{1}$
}

\author{
Iskra Pavez Soto*
}

\begin{abstract}
Resumen
El objetivo de este artículo es reflexionar sobre el concepto de infancia en la sociología en general -mediante el análisis de las ideas de socialización en sociólogos clásicos y contemporáneos-, y las principales premisas teóricas de un campo de estudios emergente denominado "Sociología de la Infancia". Esta perspectiva comprende a la niñez como una categoría permanente en nuestras sociedades -aunque sus miembros se renueven constantemente- y como un fenómeno socialmente construido y variable en términos históricos. Además, se ve a las niñas y los niños como actores sociales que participan de diversas maneras en la vida social, aunque de forma diferente a las personas adultas, tal vez por eso no siempre su acción social es visible para toda la sociedad.
\end{abstract}

Palabras clave: Sociología - infancia - acción social.

\begin{abstract}
The aim of this article is to explore the concept of childhood in sociology in general-by analyzing the ideas of socialization in contemporary-classical sociologists- and major theoretical premises of an emerging field of study called "Sociology of Childhood". This perspective understands childhood as a permanent category in our societies -although its members are constantly renewed-and as a socially constructed phenomenon and historically variable. In addition, girls and boys are seen as social actors involved in different ways in social life, although differently from adults, perhaps because of that their social action is not always visible to the whole society.
\end{abstract}

Keywords: Sociology - childhood - social action.

1 Una versión anterior de este artículo constituye el Capítulo II -denominado "Sociología de la Infancia"- el cual forma parte del marco teórico de la Tesis Doctoral de Sociología de la autora titulada: "Migración infantil: rupturas generacionales y de género. Las niñas peruanas en Barcelona y Santiago de Chile" (Universidad Autónoma de Barcelona, 2011).

* Doctora en Sociología, Universidad Autónoma de Barcelona. Trabajadora Social, Universidad Tecnológica Metropolitana. Integrante fundadora del "Grupo de Sociología de la Infancia y la Adolescencia de España", dirigido por la Doctora Lourdes Gaitán. Actualmente es Directora de la Carrera de Trabajo Social de la Universidad Alberto Hurtado. 


\section{INTRODUCCIÓN}

Durante el siglo XX se aprecia un aumento en la preocupación por las condiciones de vida de la niñez, sensibilidad que se plasma en la promulgación por parte de la ONU en 1989 de la Convención Internacional de los Derechos del Niño (en adelante CDN o Convención). Si bien es evidente la creciente protección que la sociedad despliega sobre las niñas y los niños, en la práctica se observan ciertas contradicciones en el trato que éstos reciben ya que no se permite su plena participación en la sociedad donde viven ni se consideran sus opiniones en los asuntos que les afectan. En este mismo período histórico, de modo particular en la sociología, son escasos los trabajos publicados que expresan un genuino interés por la infancia en tanto categoría social.

Los conceptos de infancia y niñez presentan una gran complejidad no sólo a nivel social, sino desde la propia etimología de estas palabras. Según la Real Academia de la Lengua Española (RAE), "Infancia" proviene del latín infantia, cuyo significado primario alude a la incapacidad de hablar y define a los înfâns o înfantis como aquellos que no tienen voz. Para la RAE actualmente la infancia es delimitada como: i) el período de la vida humana desde que se nace hasta la pubertad; ii) el conjunto de los niños de tal edad; y iii) el primer estado de una cosa después de su nacimiento o fundación. Por otro lado, la RAE define al infante como: i) el niño que aún no ha llegado a la edad de siete años; ii) el pariente del rey que por gracia real obtiene el título de infante o infanta; y iii) cada uno de los hijos varones y legítimos del rey, nacidos después del príncipe o de la princesa.

Wasserman (2001: 61) resalta que la etimología de la palabra infancia proviene del latín in-fandus, que significa no habla o que no es legítimo para tener la palabra. Ella menciona que se le llame "infante" al hijo del rey que está en la línea sucesoria al trono y que no puede ser heredero mientras el primogénito -heredero de hecho- esté vivo. Por lo tanto, su etimología expresa claramente que la palabra infancia refiere más bien a quienes no tienen permitido hablar, y no tanto sobre quienes carecen de este atributo por los años de edad (Wasserman, 2001: 61).

Según la RAE, la palabra niño o niña proviene de la voz infantil o la expresión onomatopéyica ninno, que refiere al que está en la niñez, que tiene pocos años, que tiene poca experiencia o que obra con poca reflexión y advertencia, entre otras características. Por otra parte, la niñez es definida por la RAE como: i) el período de la vida humana que se extiende desde el nacimiento a la pubertad -mismo significado de la palabra infancia-; b) el principio o primer tiempo de cualquier cosa; y c) la niñería, acción propia de niños. Tanto la palabra infancia como niñez sugieren el período vital que transcurre durante los primeros años de la vida humana. Para Wasserman (2001: 61) la palabra niño o niña presenta una polisemia muy amplia, aunque en general alude a la condición de las personas con pocos años de edad o que se encuentran en posición de subordinación social (servidumbre). La palabra niña también se utiliza para designar a la pupila del ojo, ya que "pupila" proviene del latín pupilla que significa huérfana menor de edad y que está bajo la autoridad de una persona tutora².

2 Durante la época del Imperio Romano se establecía que la infantia duraba desde el nacimiento hasta los siete años; luego desde los siete hasta los doce años en el caso de las niñas y hasta los catorce para los niños 
Desde el punto de vista de la sociología, Gaitán (2006b) define que la infancia sería una condición social delimitada por una construcción cultural e histórica diferenciada y caracterizada por relaciones de poder, mientras que las niñas y los niños serían el grupo de personas o sujetos sociales que se desenvuelven en dicho espacio social. Para efectos del presente artículo se entenderá que la infancia es un espacio socialmente construido -tal como se revisará este concepto en detalle más adelante--, mientras que la niñez se entiende como el grupo social que conforman las niñas y los niños.

En primer lugar presentamos una breve revisión sobre cómo ha sido comprendida la infancia en la sociología, analizando críticamente el concepto de infancia en sociólogos clásicos como Durkheim y Parsons, y algunos contemporáneos como Berger y Luckmann. También se muestran las principales premisas teóricas de la denominada "Sociología de la Infancia" a través de la revisión del enfoque estructural, constructivista y relacional, según la clasificación hecha por Gaitán (2006b). Por último, el artículo concluye rescatando los principales aportes y desafíos de esta perspectiva en el estudio de la niñez moderna.

\section{DE LA INFANCIA EN LA SOCIOLOGÍA A UNA SOCIOLOGÍA DE LA INFANCIA}

La infancia, como unidad de análisis, continúa siendo un objeto de difícil investigación en la sociología, ya que generalmente su estudio ha formado parte de las investigaciones en torno a la familia -como institución social- o la educación -como instrumento de reproducción del orden social a través de la dominación de las nuevas generaciones-. Según Gaitán (2006b: 15) y Rodríguez (2007: 22), los estudios sociológicos se han ocupado indirectamente de las niñas y los niños solamente de un modo instrumental. La "Sociología de la Infancia" comparte intereses teóricos y metodológicos con la "Sociología de la Juventud" - o con una sociología basada en el estudio de las generaciones-, y principalmente forma parte de los denominados childhoods studies o "estudios de infancias", un campo de estudios multidisciplinar en el que participan diversas disciplinas como la sociología, historia, antropología, trabajo social o geografía, entre otras (Qvortrup et al., 2009).

\subsection{La infancia en los enfoques sociológicos clásicos}

Debido a la situación de dependencia y a la interpretación que se ha hecho del desarrollo biopsicosocial en que se halla la niñez en el mundo contemporáneo, ésta ha sido considerada una etapa o situación presocial; es decir, como una fase de preparación para la vida adulta, la cual se considera verdaderamente vida social. Se cree que las niñas y los niños están en tránsito para ser integrados plenamente en la sociedad, una vez que dejen

correspondía la pueritia. Posteriormente venía la adulescentia de los doce o catorce hasta los 21 años de edad; a partir de ese momento se entra en la etapa de la plenitud denominada iuventus, la cual dura hasta los cincuenta años. Desde los cincuenta años y hasta los 72 se denomina gravitas, y a partir de los 72 años hasta la muerte, senectus (Wasserman, 2001). 
atrás las características propias de la infancia y dejen de ser vistos como seres infantilizados (Qvortrup, 1987) $)^{3}$.

En esta línea de argumentos podemos ubicar a Émile Durkheim, quien interesado en la Sociología de la Educación desarrolla un pensamiento sobre la infancia como un fenómeno presocial: "Un terreno casi virgen donde se debe construir partiendo de la nada y poner en ese lugar una vida moral y social" (Durkheim, 1975: 54). Este autor plantea la necesidad de una pedagogía moral que eduque y supere la supuesta naturaleza "salvaje" del sujeto infantil. En este enfoque las niñas y los niños son vistos como "receptáculos" vacíos del accionar adulto (la idea de la tabula rasa) y, por lo tanto, se justifica la necesidad de controlar esa naturaleza "salvaje" a través del poder civilizatorio de la educación escolar".

El hecho de asociar la infancia a un estado más cercano a la naturaleza que a la cultura -al igual que ocurre con otras minorías políticas, como las mujeres o los grupos indígenasconstruye un estereotipo generacional sobre las niñas y los niños como seres inferiores, que necesariamente deben estar situados bajo el poder y la autoridad de una persona adulta, la cual se considera racional y civilizada. En este enfoque se evidencia una preocupación por los mecanismos sociales por los cuales las niñas y los niños "dejan de serlo"; es decir, el proceso mediante el cual se convierten en personas adultas educadas, civilizadas y no infantiles. Por lo tanto, el interés está puesto más en el resultado que en el proceso escolar y en las relaciones de poder que se construyen entre los actores que participan de él. Durkheim considera a la infancia como un período de crecimiento en sentido físico y moral, como el período en el que la persona está preparándose para entrar a vivir la verdadera vida social: la adulta (Qvortrup, 1992, 1994a; Wintersberger, 1994; Gaitán, 2006b: 47; Rodríguez, 2007: 33 y ss.). Según Franzé (2002: 202, 321), tradicionalmente la educación escolar ha considerado este concepto de niñez para situarse como la disciplina "experta" en la infancia moderna, cuya capacidad de actuación se resume en un discurso escolarizante y civilizatorio sobre los sujetos que supuestamente carecen de las normas básicas para convivir en la sociedad ${ }^{5}$.

3 Jean Jacques Rousseau (1762) sostiene que las niñas y los niños son comparables a su idea sobre el mito del "buen salvaje" porque se desarrollarán de acuerdo con el plan de la naturaleza y que el adoctrinamiento adulto podría llegar a corromperles. Este autor propone cuatro estadios de desarrollo que van desde la infancia, niñez, niñez tardía y adolescencia (Rice, 1991: 130; Moscoso, 2008: 269).

4 John Locke decía que las niñas y los niños son una tabula rasa, es decir, un papel en blanco. Este autor pensaba que la familia puede moldearlos a través de las imitaciones, repeticiones, recompensas y castigos. Sugería a las madres y padres premiar las buenas acciones con alabanzas y aprobación y desaconsejaba el castigo físico porque no fomenta el autocontrol. Este autor fue uno de los pioneros del conductismo moderno (Rice, 1991: 130). Franzé (2002) revisa la institución educativa desde la construcción social de la infancia en términos de origen nacional o pertenencia étnica, particularmente a través de una etnografía con niñas y niños inmigrantes en escuelas públicas de Madrid. Señala Franzé (2002: 202 y ss.) que "en el imaginario occidental el niño es una criatura en transición hacia la adultez, ser incompleto convertido en objeto de la educación formal o informal, la educación civilizatoria. No es casual la vieja hermandad establecida entre la infancia y el primitivismo (Ariés) ya sea que se conciba como un estado perverso de los humanos (Freud) o como ingenuidad y bondad primigenia (Rousseau). El lenguaje escolar -y no sólo el escolar- evoca tales hermandades toda vez que se descubren categorías de niños "resistentes" a las sanciones disciplinarias. Se los piensa como seres especialmente incompletos (inmaduros, faltos de personalidad). Ellos son como unos "salvajes". Más que nada, cuerpos cuyas fuerzas incontroladas se resisten a la disciplina, cercanos a una suerte de estado de naturaleza en tanto rebeldes a la privatización de las manifestaciones corporales". 
Los estudios de Durkheim influyeron en las ideas de Talcott Parsons (1959), de modo particular en los estudios sobre los procesos de socialización que ocurren dentro de las familias (heterosexuales y nucleares); es decir, "en el proceso de adquisición por parte de los niños de las pautas y los roles sociales" (Parsons, 1959: 200). Este autor sostiene que toda primera y normal socialización infantil debe producirse dentro de la familia, con la complementariedad de otras agencias como la escuela y el grupo de iguales, aunque recalca que la socialización es diferenciada en virtud del género, la clase social y el grupo étnico (Parsons, 1959: 218) ${ }^{6}$.

Chris Jenks (1996) se ha dedicado a analizar el interés de Parsons en el proceso de socialización en que participa la niñez, entendido como la reproducción social entre las generaciones. Según Jenks (1996), a Parsons lo que verdaderamente le inquieta no es la infancia en sí misma -como categoría sociológica-, sino el proceso de socialización. En la teoría funcionalista de Parsons las niñas y los niños son vistos como receptores pasivos de los contenidos que la sociedad considera necesarios para que una persona se convierta en adulta. Se trata de los mecanismos que reproducen el orden social en el seno de una sociedad funcionalista. Desde el momento del nacimiento -e incluso antes del nacimiento, en términos de género- se depositan demandas y exigencias a través de la asignación de un determinado rol a las niñas y los niños. En la mirada funcionalista la figura infantil encarna ese "Yo" social que participa en el proceso de socialización, cuyo objetivo principal es transformar un ser infantil "salvaje" en un producto social, que no es otra cosa que una persona adulta normalizada. En este enfoque se piensa a las niñas y los niños desde la metáfora de un objeto "esponja" que recibe pasivamente dicha socialización. Sin embargo, el individuo no sólo reproduce ese orden social, también lo recrea, lo innova e incluso lo subvierte. En cada contexto histórico y territorial las niñas y los niños despliegan diversas formas de vivir sus infancias, por lo tanto los modos en que se reproduce el orden social también cambian. El movimiento socializador es bidireccional y no sólo unidireccional desde la persona adulta hacia las niñas y los niños (Rodríguez, 2000; Gaitán, 2006b: 46).

\subsection{La participación infantil en el proceso de socialización}

En la visión funcionalista, la infancia representa el momento de entrada e incorporación de la niña o el niño a su cultura, un proceso adaptativo similar al que viven, por ejemplo, las personas que participan en procesos migratorios. Pero, en la visión parsoniana, el sujeto infantil viene limpio de experiencias culturales anteriores y, por lo tanto, puede ser modelado e inculcado en las pautas y valores que más convienen a la sociedad. El sistema social actúa y constriñe al nuevo ser en función del orden, la integración y la corrección esperada (Parsons, 1959). La socialización, así entendida, se caracteriza por la plasticidad, sensibilidad y dependencia infantil, ya que la niña o el niño desempeña el rol de ser "socializado" por un "socializador". En esta teoría la niñez es esencialmente pasiva y evaluada en términos

6 Parsons (1959:202) señala que la niña y el niño realizan su aprendizaje de los roles y pautas sociales a través de la imitación de un modelo adulto o la identificación y la instrucción que este modelo ejerce. Es decir, un proceso unidireccional y esencialmente pasivo desde el punto de vista infantil. 
evolutivos: el individuo está en fase de crecimiento para alcanzar el estado deseable (adultez) en cuyo momento será integrado en la sociedad, porque se piensa que cuando las niñas y los niños están en la fase infantil no forman parte de la sociedad adulta, sino que habitan un mundo paralelo al de ésta (Gaitán, 1999a: 115-118).

Parsons (1959: 203) se refiere al poder que tiene la persona adulta de dar recompensas o castigos mientras está instruyendo a las niñas y los niños en el refuerzo o extinción de determinados actos (condicionamiento), a través de lo que este autor denomina mecanismos de socialización. El autor describe el rol que juegan estos mecanismos en el aprendizaje de las normas y los roles sociales, pero no analiza la dimensión del poder generacional que emana en el hecho de conceder recompensas e infligir castigos. En la teoría de Parsons, los castigos forman parte del "necesario" proceso de socialización.

Sin embargo, desde una mirada crítica, la facultad de imponer castigos representa la expresión del poder que tienen las personas adultas, ya que éstas están situadas en una posición de dominación y autoridad sobre las niñas y los niños. Tal y como señala Foucault (1976: 183, 185), en todos los sistemas disciplinarios existen mecanismos para penalizar con castigo físico o pequeñas humillaciones las conductas que se consideran inadecuadas en el proceso de adaptación al propio sistema. En este caso, los castigos que reciben las niñas y los niños serían una forma de control social que se ejerce al interior de la propia familia o la escuela en tanto instituciones disciplinarias, es decir, sistemas basados en poder y normas que transmiten el "deber ser" en la sociedad. Además, los castigos y las sanciones que reciben las niñas y los niños están diferenciados en términos de género.

El aprendizaje del "deber ser" no sólo es una cuestión generacional, es decir, es la obediencia que deben mostrar las niñas y los niños hacia las normas que les enseñan las personas adultas. Se trata de normas establecidas en términos de género, ya que justamente es durante la infancia cuando se acelera e intensifica el proceso de aprendizaje de los roles de género y las relaciones de poder entre los géneros. En la mirada funcionalista se espera e incentiva a que las niñas y los niños obedezcan a las personas adultas (madres, padres y docentes) porque ellas realizan la función de socializar a las nuevas generaciones, es decir, tienen una autoridad para hacer cumplir con la reproducción del sistema social (Mayall, 2000, 2002).

En este sentido, según Jenks (1996) el enfoque de la socialización de la infancia asume algunos supuestos de las teorías del desarrollo biológico, aunque sin cuestionarlos desde una mirada sociológica. Los estudios de Sigmund Freud (1970), Erik H. Erikson (1970) y Jean Piaget $(1972,1977)$ han tenido una gran influencia en la visión funcionalista de la infancia, en la cual se reduce la complejidad de este fenómeno social sólo como un período de desarrollo biológico y una etapa de socialización. La niñez aparece así como un momento en el cual se conectan la dimensión individual y social de un individuo, donde se aprenden las normas morales, se internalizan los roles sociales y se desarrollan las habilidades cognitivas. En consecuencia, se yergue como la etapa privilegiada de reproducción del orden social establecido mediante la socialización de las nuevas generaciones. Piaget $(1972,1977)$ estudia a las niñas y los niños principalmente a nivel individual (the child) -del mismo modo que se 
pretende estudiar a "la mujer" o "el indígena" partiendo de una supuesta esencia universal en cada uno de estos sujetos-, mientras que el objetivo de la Sociología de la Infancia es intentar mirar la niñez como un fenómeno social y ver a las niñas y los niños como un grupo social des-naturalizado de una esencia infantil universal concebida a priori (James y Prout, 1997: 11 y ss.). Lo anterior se refleja muy bien en la siguiente cita:

“...the criticism of a psychology based on universal laws that were supposed to hold good across all societies and at all historical times. It was argued that such terms as 'the mother' and 'the child' not only conveyed a meaningless generality but also misrepresented the relationship between individual and social worlds and portrayed social arrangements as if they were fixed by laws of nature" (Richard, 1986: 3; citado en James y Prout, 1997: 20).

Si bien es cierto que ocurren fenómenos biológicos durante la infancia, también es cierto que durante toda nuestra existencia nuestros cuerpos se ven afectados y modificados por las condiciones materiales, sociales, económicas y culturales en las que vivimos. El enfoque sociológico que aquí se presenta intenta cuestionar la supuesta universalidad del desarrollo biológico infantil e intenta visibilizar las condiciones sociales que inciden y determinan igualmente dicho proceso. James y Prout (1997: 21) argumentan que algunos movimientos sociales -tales como los que promueven los derechos civiles para las personas afroamericanas o los feministas- han sugerido que las relaciones sociales no se forman a partir de leyes psicológicas o biológicas universales, sino, muy por el contrario, han sido acordadas políticamente a través de la historia. Pero, para el caso particular de las niñas, los niños y la infancia, hasta ahora no han sido cuestionadas desde categorías sociológicas y políticas de exclusión, salvo parcialmente en el debate sobre la adecuación legislativa tras la promulgación de la Convención Internacional de los Derechos del Niño de la ONU (1989). Chris Jenks (1996: 4) lo expresa de la siguiente forma:

\footnotetext{
"The history of the social sciences has attested to a sequential critical address and debunking of the dominant ideologies of capitalism in relation to social class, colonialism in relation to race, and patriarchy in relation to gender; but as yet the ideology of development in relation to childhood as remained relatively intact".
}

El enfoque de la infancia como un momento de desarrollo biológico es necesario y no se niega su existencia, pero no es suficiente para comprender todas las dimensiones del fenómeno social de la niñez contemporánea. Por su parte, Mayall (2002) indica que la visión sobre el desarrollo biológico infantil está basada en presupuestos acerca de los roles de género normativos y deseables en cada sociedad. No obstante, dicho desarrollo biológico de las niñas y los niños ocurre en determinados contextos de clase social, género o pertenencia étnica, es decir, es producido por las propias condiciones sociales en que viven los sujetos infantiles (Mayall, 2000, 2002).

Tal y como sostienen Gaitán (2006b: 50) y Rodríguez (2007: 54), estudiar la situación de vida de las niñas y los niños considerándolos como "potenciales adultos" nos lleva a interesarnos únicamente por las consecuencias futuras que tendrán las condiciones de vida presentes, restando importancia al impacto de éstas en el momento presente de la vida infantil. Qvortrup (1992: 176) lo expresa así: "la idea de que importa el futuro de los niños 
es peligrosa desde un punto de vista ético, porque mide el bienestar del niño basándose en su vida futura y no en su bienestar presente. Esta opinión implica no estar interesado en el niño, sino, en los adultos". Para James y Prout (1997: 7) la infancia no es sólo una fase de desarrollo y el tránsito por las etapas vitales, sino que también constituye un producto social en el que tal desarrollo ha de materializarse:

"The immaturity of children is a biological fact of life but the ways in which this immaturity is understood and made meaningful is a fact of culture (...). It is these 'facts of culture' which may vary and which can be said to make of childhood a social institution. It is in this sense, therefore, that one can talk of the social construction of childhood and also, of its re- and deconstruction. In this double sense, then, childhood is both constructed and reconstructed both for children and by children" (James y Prout, 1997: 7).

Para Qvortrup (1992: 177), en la psicología "existe una idea de ascenso gradual en sentido cualitativo, el paso de estados de imperfección a estados de perfección, de inmadurez a madurez, de incapacidad a capacidad, de irresponsabilidad a responsabilidad". Pero, ¿qué tiene por decir la sociología y otras ciencias sociales respecto a las dimensiones sociales, políticas, culturales y económicas del fenómeno de la infancia? La constante alusión al futuro y la consideración de las niñas y los niños como un "proyecto de persona" o becoming, pero sin serlo totalmente en la vida presente, ha tenido como consecuencia su exclusión de la vida social y pública. Además, según James y Prout (1997: x, 9) la visión biologicista -para explicar fenómenos sociológicos- puede considerarse obsoleta en otras áreas de las ciencias sociales, como por ejemplo en los estudios culturales o los estudios de género, pero en la interpretación sociológica de la infancia continúa vigente sin cuestionarse del todo. La Tabla 1 muestra algunos de los presupuestos que están en la base de la visión sociológica que posee un sesgo reduccionista de la infancia, entendida sólo como una fase de socialización y una etapa de desarrollo biológico ignorando que se trata de un fenómeno social complejo con múltiples dimensiones (James y Prout, 1997: 13).

Es difícil sostener empíricamente que las personas adultas están completas y definitivas sin capacidad de cambiar porque permanentemente experimentan la influencia de las estructuras y las relaciones sociales en las que viven y, por lo tanto, poseen la capacidad de transformarse y también de modificar los entornos que habitan. Durante toda nuestra existencia tenemos la capacidad de aprender, y de hecho seguimos aprendiendo normas u otros roles sociales, y esto porque nuestras habilidades cognitivas y sociales siempre están en desarrollo. Actualmente, en el mundo globalizado en el que vivimos, las personas adultas deben necesariamente demostrar su capacidad de aprendizaje de nuevas tecnologías, idiomas y conocimientos que se requieren para enfrentar la vida cotidiana (desde la electrónica hasta internet). Por lo tanto, comprender a la infancia en términos sociológicos también nos ayuda a cuestionar el concepto preconcebido sobre la adultez y las otras generaciones.

Precisamente, los estereotipos generacionales significan ideas preconcebidas y exigencias normativas que recaen sobre las personas cuando se las predetermina a partir de la edad. Al igual que ocurre con el género, la clase social o la pertenencia étnica, la edad es una categoría social en nuestra sociedad que implica una determinada categorización etaria con derechos 
TABLA 1

Visión sociológica reduccionista de la infancia

\begin{tabular}{|c|c|c|}
\hline CONCEPTOS & SOCIALIZACIÓN & DESARROLLO BIOLÓGICO \\
\hline Infancia & $\begin{array}{l}\text { Una fase preparatoria para la vida } \\
\text { adulta; en esta última es donde } \\
\text { realmente se participa en la vida } \\
\text { social. }\end{array}$ & $\begin{array}{l}\text { Una etapa del desarrollo evolutivo } \\
\text { del ser humano hacia la adultez; es } \\
\text { esta última la que se valora como } \\
\text { plena y deseable. }\end{array}$ \\
\hline \multirow[t]{2}{*}{ Las niñas y los niños } & $\begin{array}{l}\text { Meros receptores pasivos del orden } \\
\text { social ("receptáculos" y "esponjas"). }\end{array}$ & $\begin{array}{l}\text { Incapaces, inmaduros e inacabados } \\
\text { en comparación con las personas } \\
\text { adultas, a quienes se supone } \\
\text { capaces, maduras y acabadas. }\end{array}$ \\
\hline & Seres presociales. & $\begin{array}{l}\text { Seres biológicos, organismos } \\
\text { respondientes. }\end{array}$ \\
\hline Interés & $\begin{array}{l}\text { Visión centrada en el producto } \\
\text { social del proceso de socialización; } \\
\text { importa la persona adulta } \\
\text { normalizada en que se convertirá el } \\
\text { ser infantil. }\end{array}$ & $\begin{array}{l}\text { Visión centrada en el resultado (ser } \\
\text { adulto) del proceso de desarrollo; } \\
\text { una vez que la persona "acabe" el } \\
\text { proceso, será completa. }\end{array}$ \\
\hline Autores de referencia & Parsons & Durkheim, Piaget \\
\hline
\end{tabular}

Fuente: Elaboración propia en base a James y Prout (1997: 11 y ss.), Gaitán (2006b: 45 y ss.) y Rodríguez (2007: 54 y ss.).

y deberes que van más allá de las leyes vigentes, porque forman parte de las representaciones sociales de la edad y la generación. Del mismo modo que los estudios feministas ofrecen una reflexión sobre los estereotipos de género al deconstruir las exigencias y los mandatos que recaen normativamente sobre las mujeres y los hombres por su condición de género, la Sociología de la Infancia nos permite analizar los estereotipos generacionales atribuidos normativa y arbitrariamente a las personas en función de su edad. Desde la Sociología de la Juventud (Duarte, 2001), la categorización por edades supone ciertos ritos de paso que deben ser cumplidos en cada etapa para ingresar a la siguiente, de manera ascendente y gradual, hasta llegar al estadio deseable que es la adultez; los sujetos que no cumplen con dichas demandas y exigencias según cada edad son excluidos de dicha categoría. Lo anterior se aplica en el estudio de la infancia respecto a las exigencias normativas que recaen sobre las niñas y los niños cuando se evalúa permanentemente la edad en la cual se debe aprender a caminar, a controlar esfínteres, a leer, a escribir... sin considerar los contextos sociales donde ocurren dichos procesos. 


\subsection{La infancia en los enfoques sociológicos contemporáneos}

En la teoría sociológica contemporánea existen autores que analizan la situación de la infancia en la sociedad como un hecho eminentemente social, y representan un primer intento en la consideración de la infancia como objeto de interés sociológico autónomo. El enfoque del interaccionismo simbólico de George H. Mead (1982) ofrece una mirada más matizada sobre el papel que juegan las niñas y los niños en el proceso de internalización de lo social. Este autor sostiene que el sujeto infantil está en permanente diálogo consigo mismo y con los otros (el otro generalizado) que le rodean, lo que se lleva a la práctica a través del juego espontáneo y el juego organizado. En este caso se considera que la vida de los individuos es un fenómeno social desde el nacimiento y, por lo tanto, toda la vida social está afectada por las fuerzas y el poder de la estructura social. El individuo, incluso siendo niña o niño, siempre tiene un cierto grado de autonomía y una subjetividad propia (Rodríguez, 2007: 40 y ss.).

Por su parte, el enfoque constructivista de Berger y Luckmann (1968) también es clave en esta nueva mirada del sujeto infantil en la sociología. Estos autores señalan que el ser humano es un producto social y todo su desarrollo está socialmente construido e interferido, incluso la infancia. Para estos autores, en la construcción social de la realidad se registran los momentos de externalización, objetivación e internalización a través del proceso de socialización primaria y secundaria donde se internaliza la realidad objetiva. La socialización primaria se lleva a cabo especialmente en la familia y funciona por pautas más emocionales que racionales, a la vez que mediatizada por un fuerte control social: cómo debo ser y qué debo hacer, a través de la identificación de los otros significantes. La socialización secundaria, en cambio, se desarrolla en ciertas instituciones destinadas para este fin, como la escuela, la iglesia, el ejército, etc. La socialización requiere ciertas imposiciones a las niñas y los niños para su amoldamiento social, lo que trae como consecuencia la adjudicación de un lugar en el mundo social. Así lo resumen los autores:

“aunque el niño no sea un mero espectador pasivo en el proceso de su socialización, son los adultos quienes disponen las reglas del juego. El niño puede intervenir en el juego con entusiasmo o con hosca resistencia, pero por desgracia no existe ningún otro juego a mano" (Berger y Luckmann, 1968: 171).

Si la socialización transmite la experiencia en el lenguaje, las niñas y los niños recrean, transmiten y reconstruyen la realidad que les ha sido dada, configurando su propia cultura infantil; pero son las personas adultas quienes imponen el orden social que debe reproducirse (Gaitán, 2006a: 33). Por otro lado, Norbert Elías (1989) identifica que en las sociedades modernas el proceso civilizatorio se plasma a través de la educación y otras instituciones de poder y control-como el Estado- que ejercen una verdadera presión generacional sobre la infancia. El resultado es que el mundo infantil queda relegado al ámbito privado, familiar y escolar, como una etapa "preparatoria" para la vida adulta y pública, que es cuando se espera que la persona haya alcanzado cierto grado de civilización (autocontrol, obediencia, rutina de trabajo, etc.), lo que se denomina "madurez". La principal aportación de los estudios sociológicos contemporáneos es la de subrayar la importancia de la experiencia social en 
la infancia como fuente para el surgimiento del agente social capaz y autónomo, alejado de la imagen del organismo respondiente (Rodríguez, 2007: 44). Considerar el desarrollo y la socialización infantil en un contexto social es el primer paso para pensar la infancia como un fenómeno social (James y Prout, 1997: 24).

\subsection{Antecedentes de la Sociología de la Infancia}

Durante las décadas de los años ochenta y noventa se publican diversos trabajos sociológicos -particularmente en el ámbito académico anglosajón (Europa y Estados Unidos) sobre la necesidad de revisar el concepto de infancia en la sociología contemporánea. Estas publicaciones conforman las bases teóricas de la Sociología de la Infancia, pero lamentablemente la mayoría de ellas no han sido traducidas al castellano y tal vez por esta razón permanecen desconocidas o marginales en los debates sociológicos hispanoamericanos.

En España, alrededor del año 2000 comienzan a surgir algunos artículos y libros publicados principalmente por Lourdes Gaitán (1999b, 2006a, 2006b, 2008) e Iván Rodríguez (2000, 2007), quienes realizan la importante tarea de articular los enfoques teóricos de la tradición anglosajona y contextualizarlos en el debate sociológico español. Durante este mismo tiempo, en América Latina se publican algunos trabajos académicos sobre la Sociología de la Infancia de José Sánchez-Parga (2004), René Unda (2003, 2009) y el Instituto de Formación de Educadores de Jóvenes, Adolescentes y Niños Trabajadores (IFEJANT) (2003).

En Chile paulatinamente algunas autoras (Vergara et al., 2010; Pavez, 2010, 2011a, 2011b, 2012) comienzan a utilizar el enfoque de la Sociología de la Infancia para estudiar el fenómeno de la niñez contemporánea, pero todavía existe un gran desconocimiento de esta perspectiva en nuestro país, por ello la bibliografía que se utiliza en este artículo es principalmente de origen europeo y estadounidense.

Uno de los trabajos pioneros en el ámbito anglosajón lo constituye la obra de Chris Jenks titulada The Sociology of Childhood, publicada en 1982. En esta obra el autor revisa el concepto de infancia en los trabajos de Parsons, Durkheim y Piaget, entre otros (tal como vimos anteriormente), para demostrar que cada modelo teórico de vida social da lugar a una diversidad de visiones de la infancia, lo que demuestra su construcción social. Posteriormente, en 1987 Jens Qvortrup coordina un número monográfico de la International Journal of Sociology dedicado a la Sociología de la Infancia, declarando en la introducción de este trabajo lo siguiente:

"el postulado de que la infancia (como un elemento estructural y como una posición de estatus) cambia en tiempo y espacio de acuerdo con las necesidades e intereses de la sociedad adulta dominante parece tan evidente, incluso casi trivial, que podemos sorprendernos de que sea casi imposible encontrar esta perspectiva representada entre los sociólogos" (Qvortrup, 1987: 6, negrita nuestra).

En el mismo año 1987 Qvortrup asume la dirección del proyecto de investigación titulado "La infancia como fenómeno social" en el marco del Programa de Infancia del Centro Europeo para el Bienestar Social de Viena, un estudio aplicado a 16 países europeos (Qvortrup et al., 
1994) ${ }^{7}$. Dicha investigación asume un enfoque teórico sociológico -no sólo psicológico o educativo- para demostrar la posibilidad de investigar el fenómeno de la infancia en cada sociedad a partir de la edad como categoría de análisis. En 1990 la International Sociological Association autoriza el establecimiento de un grupo temático denominado Sociology of Childhood, esto sucede un siglo después que se crearan similares grupos expertos en disciplinas como la psicología, psiquiatría o medicina (Qvortrup, 1994a: x). Los trabajos realizados por el Centro Europeo de Viena sientan las bases teóricas del enfoque estructural que junto al constructivista y el relacional conforman los tres enfoques de la Sociología de la Infancia de acuerdo a la clasificación hecha por Gaitán (2006a, 2006b).

\subsection{Enfoque estructural de la Sociología de la Infancia}

El enfoque estructural de la Sociología de la Infancia, tal como su nombre lo indica, intenta analizar la posición de la niñez en la estructura de las sociedades. La perspectiva estructural parte de la premisa de que la infancia como categoría existe permanentemente en la sociedad, aunque sus miembros se renueven constantemente -tal como ocurre con la juventud-. Por lo tanto, desde esta perspectiva las condiciones de la vida infantil se analizan considerando el nicho social que ocupa la niñez de ahora y la que vendrá, como un espacio en la estructura de la sociedad que está destinado a que lo habiten las personas cuando son niñas y niños. En otras palabras, se piensa a la infancia en términos sociológicos como un grupo social en permanente conflicto y negociación con los otros grupos sociales y no tanto a nivel individual, como lo hacen las disciplinas psicológicas o pedagógicas (Qvortrup, 1992, 1994b; Wintersberger, 1994). La población infantil se entiende como el "colectivo de individuos muy diferentes entre sí, pero que comparten una misma ubicación etaria en tanto 'menores de edad' sometidos a la autoridad adulta" (Rodríguez, 2007:56).

Tal como ya se dijera, el proyecto de investigación del Centro Europeo de Viena, a partir de los resultados de su mencionado estudio, obtiene 16 informes nacionales sobre la situación de la infancia en cada país. De ello derivan las nueve tesis acerca de la "infancia como un fenómeno social" que sientan las bases teóricas del enfoque estructural de la Sociología de la Infancia (Gaitán, 1999a: 128). Estas nueve tesis son las siguientes:

1. La infancia es una forma particular y distinta de la estructura social de cualquier sociedad.

2. La infancia es, sociológicamente hablando, no una fase transitoria, sino una categoría social permanente.

3. La idea de niña o niño como tal es problemática, mientras que la infancia es una categoría variable histórica y social.

$7 \quad$ En el marco de este estudio se desarrolla en Madrid en julio de 1991 el "Seminario Europeo sobre Investigación y Políticas de Infancia en Europa en los años 90". En ese contexto se elaboran algunos de los escasos documentos en español de Qvortrup (1994) y Wintersberger (1994), dos brillantes exponentes de este enfoque (Gaitán, 2006b: 51). 
4. La infancia es una parte integral de la sociedad y su división del trabajo.

5. Las niñas y los niños son ellos mismos coconstructores de la infancia y la sociedad.

6. La infancia está expuesta en principio a las mismas fuerzas que las personas adultas (económica, institucionalmente, por ejemplo) aunque de modo particular.

7. La dependencia estipulada en las niñas y los niños tiene consecuencias para su invisibilidad en las descripciones históricas y sociales.

8. No las madres y los padres, sino la ideología de la familia, constituye una barrera contra los intereses y el bienestar de las niñas y los niños.

9. La infancia tiene la categoría de una minoría clásica, que es sujeto de tendencias de marginalización y paternalización.

Las dimensiones de análisis que autores como Qvortrup o Wintersberger consideran claves en el estudio estructural de la infancia son: los cambios demográficos; las actividades infantiles; la economía; la justicia distributiva y el estatus jurídico. Habría que añadir que al interior de cada uno de estos focos de estudio de la dimensión estructural de la infancia existen desigualdades de género, de clase social y de origen étnico entre las niñas y los niños, en tanto grupo social. Liebel (2007) considera que en las sociedades occidentales modernas la infancia como categoría y las niñas y los niños en tanto grupo social comparten rasgos clásicos de una minoría respecto a los grupos de poder, como parte de uno de los "grupos silenciados" (muted groups):

- Un amplio número de personas comparte la condición que es causa de discriminación. En cada sociedad hay una proporción de población infantil y justamente debido a nuestra biología todos los seres humanos viven la condición infantil. Por lo tanto, la niñez es un grupo social presente en todas las sociedades (Liebel, 2007).

- La posición que ocupan en la sociedad se considera inferior, está deslegitimada y carece de estatus de reconocimiento. Es una categoría "indeseable" y que se utiliza como sinónimo de desprecio. Por ejemplo, decir que algo es "infantil" significa que es poco serio, ingenuo, inmaduro, irresponsable o irracional; la expresión "niñita" puede utilizarse como una ofensa o burla (Liebel, 2007).

- Los sujetos que comparten dicha condición son incapaces de decidir e incidir en los asuntos políticos, económicos y sociales que les afectan directamente. Las niñas y los niños no tienen capacidad de presión política, porque no tienen derecho a voto (Liebel, 2007; Gaitán y Liebel, 2011).

Por otro lado, la dependencia económica que afecta a la niñez moderna agudiza su condición de minoría respecto al poder adulto, puesto que en el sistema capitalista el hecho de obtener y disponer de dinero significa un mecanismo -aunque no el único- de empoderamiento y capacidad de tomar decisiones. Esta situación de desventaja económica Ileva a Oldman (1994: 53) a sugerir que las relaciones generacionales entre el colectivo infantil 
y el adulto puedan leerse como relaciones de clase social, cuyo estatus económico favorable lo tienen las personas adultas. La situación de dependencia económica lleva a la niñez a una subordinación y paternalización permanentes. Debido al discurso escolarizante que recae sobre la niñez moderna, las niñas y los niños no pueden trabajar remuneradamente de forma (Cordero, 2012), aunque lo deseen como una motivación para tener dinero en tanto tienen la obligación de asistir a la escuela y no reciben sueldo por el trabajo escolar que realizan en la sociedad del conocimiento (Qvortrup, 1992, 1994; Gómez-Granell et al., 2004). En este marco, las niñas y los niños trabajadores - por razones de pobreza, culturales, etc.- representan una amenaza al estereotipo de niñez deseable. Por ello se organizan acciones adultas para combatir el trabajo infantil, argumentando que son explotados laboralmente y que su deber/ derecho es estudiar (Cordero, 2012). Según Liebel (2007, 2009), en el estudio sociológico de la niñez trabajadora se articulan las categorías de clases sociales y generacionales porque el hecho de que las niñas y los niños puedan ser "productivos" significa tomar parte activa en la vida económica de sus familias y de nuestra sociedad.

\subsection{Enfoque constructivista de la Sociología de la Infancia}

Las obras de Chris Jenks (1996) y Allison James y Alan Prout (1990) se consideran fundacionales en la visión sociológica de la infancia como una construcción social, idea principal de este enfoque, el cual toma distancia crítica con el funcionalismo sociológico como marco conceptual para estudiar a la infancia. En 1990, James y Prout editan el libro titulado Constructing and Reconstructing Childhood. Contemporary Issues in the Sociological Study of Childhood; en dicha obra analizan las bases teóricas y los debates sociológicos contemporáneos que sirven de marco analítico para el estudio de la infancia en la sociología. James y Prout (1990: 8) explicitan los seis rasgos que consideran clave en la emergencia de un nuevo paradigma sociológico de la infancia:

1. La infancia es comprendida como una construcción social. Se reconoce el carácter natural (biológico) de la infancia pero integrado en un contexto social y cultural. Se comprueba que en cada sociedad aparece como un componente de la estructura y también con una dimensión cultural específica y diferente de otras sociedades.

2. La infancia es una variable del análisis social. No puede ser entendida separadamente de otras variables como el género, la clase o la etnia. Cuando se analizan estas variables interrelacionadas se comprueba que existen muchas infancias, por lo tanto, que no es un fenómeno único y universal.

3. Las relaciones sociales de las niñas y los niños son valiosas para estudiarlas por símismas, independiente de la perspectiva de las personas adultas.

4. Las niñas y los niños son y deben ser vistos como agentes; es decir, como actores sociales que participan en la construcción y determinación de sus propias vidas, de quienes les rodean y de las sociedades en que viven. Las niñas y los niños no son objetos pasivos de la estructura y los procesos sociales. 
5. La etnografía es un método particularmente útil para el estudio de la infancia, puesto que permite considerar la voz infantil en la producción de los datos sociológicos.

6. La infancia es un fenómeno en relación con la doble hermenéutica de las ciencias sociales actuales. Un nuevo paradigma sociológico sobre la infancia da cuenta de la reconstrucción social y política de la infancia en nuestras sociedades.

En este enfoque se asume que la infancia está inscrita en una estructura que afecta la vida de las niñas y los niños -tal como evidencia el enfoque estructural recién comentado-, pero se analiza la acción social de los individuos entendida como una capacidad de agencia dentro de los marcos estructurales. Para analizar la acción y la agencia infantil se toma como punto de referencia el debate sociológico sobre la estructura y la acción de los individuos propuesto por Anthony Giddens (1984). En la teoría de la estructuración de Giddens (1984) la vida social es producida por las acciones de los individuos y a través de las acciones cotidianas se va reproduciendo la propia estructura. Las estructuras inciden en las acciones y éstas a su vez están determinadas estructuralmente. Las actividades humanas conforman prácticas sociales que se denominan "praxis", ubicadas en un espacio y tiempo, es decir, en un contexto particular. En otras palabras, lo social es producto de los actores y los actores son un producto social. La acción y la estructura se refuerzan y complementan (dualidad). Por lo tanto, los actores sociales expresan su agencia al actuar en un contexto determinado que les constriñe, pero también ofrece oportunidades de nuevas acciones sociales por parte de los agentes, es decir, la capacidad de poder intervenir en una acción concreta. Si bien Giddens (1984) considera que la acción es una conducta racionalizada y reflexiva por parte del agente, es a través del lenguaje que la acción cobra significado para la propia persona y además tiene un significado socialmente construido (doble hermenéutica).

Según James y Prout (1990: 27), la sociología interpretativa ofrece la oportunidad de reexaminar el rol de las niñas y los niños como agentes activos que negocian con otros actores individuales en un marco estructural que es producido y reproducido por ellos mismos. En esta mirada se entiende que la institución de la infancia (como marco estructural) existe detrás de la actividad particular de cada niña, niño o persona adulta.

Por su parte, Mayall (2002: 21) considera que es clave comprender a las niñas y niños como actores sociales con capacidad de agencia, que contribuyen a la sociedad a través de la división del trabajo en la sociedad capitalista. Agente y actor son palabras que tienen la misma raíz latina (ago, agere, egi, actum) que se vinculan con el hacer y el actuar, pero el significado de dicha acción puede variar: un actor social tiene deseos subjetivos, mientras que el término agente sugiere negociación e interacción con otros para decidir (Mayall, 2002: 21).

La acción del agente infantil se da en el contexto estructural recién presentado, es decir, en un marco de acción minoritario (Mayall, 2002: 21). Si seguimos la idea de Berger y Luckmann (1984) respecto a que la producción y reproducción de la sociedad se desarrolla desde antes del nacimiento de las personas -por ejemplo, en términos de género- y dura toda su existencia, podemos cuestionar la visión reduccionista de la infancia entendida sólo como una etapa transitoria y fase de socialización, puesto que ambos procesos (etapa 
transitoria y socialización) forman parte de la necesaria reproducción de la propia sociedad. Esta acción se lleva a cabo a través de acciones cotidianas y permanentes que reproducen un orden generacional y de género en el que necesariamente deben participar las niñas y los niños (Qvortrup, 1992; Gaitán, 2006).

Qvortrup (1994: 3) dice que si las niñas y los niños son ontológicamente diferentes de las personas adultas, sería necesario plantear el debate en términos filosóficos sobre la verdadera ontología del ser humano. Ciertamente las personas adultas pueden ejercer poder sobre las personas infantiles y no requieren justificar su actuar porque se entiende que es un orden "natural" emanado del estatus superior adulto. Sin embargo, la capacidad de agencia de una persona se entiende como la iniciativa en la acción y en el "poder elegir" (Wartofsky, 1981: 199). Desde esta definición podemos concluir que las niñas y los niños son agentes porque actúan y construyen en su entorno, producen conocimientos y experiencias. Pero la acción infantil tiene un sentido y se desarrolla de modo distinto a la acción adulta, aunque esta última es la única que se considera legítima y por esta razón no se reconoce la praxis infantil, argumentándose la edad como el único criterio para definir competencias y capacidad de acción. Entonces, la edad viene a ser un estatus similar al sexo o la raza, una condición de los sujetos que genera relaciones sociales de poder, pero que se interpretan inherentes a dicha condición (Qvortrup, 1992).

Además de la edad, la dependencia también es considerada parte del orden natural generacional (como se consideraba la dependencia económica femenina parte del orden natural de géneros) sin cuestionarse que se trata más bien de una consecuencia de la posición de poder que ocupan las niñas y los niños (Qvortrup, 1994: 4).

Si bien se comparte la premisa estructural de que la infancia existe permanentemente en la estructura de la sociedad, en el enfoque constructivista se comprenden las especificidades de cada contexto en que se desarrolla el fenómeno de la infancia. Se entiende que la infancia no es homogénea sino que está situada y afectada por las desigualdades de género, de clase social y de origen "racial", nacional o étnico, en que participa. En cada territorio y momento histórico han sido distintas las manifestaciones del fenómeno infantil. Por lo tanto, pueden existir distintas formas de ser niña o niño, es decir, muchas infancias. De este modo se comprueba su construcción social (James y Prout, 1990: x).

Un claro ejemplo de la construcción social de la infancia es la promulgación de la Convención Internacional de los Derechos del Niño por parte de la ONU en 1989. Ciertamente, la Convención transformó el concepto moderno de infancia promoviendo una cultura más igualitaria y respetuosa de los derechos infantiles en las políticas públicas, la educación y las familias. No obstante, la Convención también recrea un determinado concepto de infancia y de las relaciones generacionales de poder que se derivan de éste al otorgar ciertos derechos y negar otros. Por ejemplo, se debate que no se concedan derechos políticos como el voto o se ignoren los derechos sexuales y reproductivos de las y los adolescentes (Gaitán y Liebel, 2011: 29).

Gaitán (2008) deduce que los derechos establecidos en la Convención representan la relación adultocéntrica que las sociedades occidentales y europeas mantienen con las 
niñas y los niños y se ha importado como un modelo universal de niñez. En este marco, son las personas adultas quienes conceden ciertas libertades, pero se mantiene el énfasis en la provisión y protección que facilitan las relaciones generacionales de poder, dando muy poca atención al ámbito de la participación.

En la misma línea crítica sobre la CDN, Alanen (1994: 28) afirma que el modelo de infancia occidental se considera universalmente válido, en parte porque algunas ciencias sociales lo han aceptado como la verdadera y normal esencia del ser infantil a través de descripciones y prescripciones. De esta manera, concluye Alanen (1994), hemos llegado a la conclusión de que el bienestar infantil deseable y medible es el modelo desarrollado en los países ricos. El concepto de infancia implícito en la Convención es característico de los países ricos y europeos, invisibilizando la enorme diversidad en la que viven las niñas y los niños alrededor del mundo (Pavez, 2011: 42). Por esta razón, Jenks (1996) habla de las "infancias" revelando que se trata de una construcción social acorde al contexto en el cual se expresa y, por lo tanto, una categoría sociológica.

\subsection{Enfoque relacional de la Sociología de la Infancia}

Las principales autoras exponentes de este enfoque son Berry Mayall $(2000,2002)$ y Leena Alanen (1994). Las autoras se proponen construir una "Sociología de los Niños" que devele el punto de vista infantil en la investigación e intervención, al considerar a la infancia como una generación con un estatus y una posición de poder determinada. Se intentan leer los procesos en que participan las niñas y los niños como relaciones generacionales de poder y negociación, similares al orden de género, constituidas también por este. Algunas de las premisas teóricas del enfoque relacional de la sociología de la infancia son las siguientes:

1. La generación es un concepto clave para entender las relaciones entre niñas-niños y personas adultas. Estas relaciones generacionales tienen una dimensión individual (microsocial) y otra social (macro) (Mayall, 2002: 1).

2. La infancia se define como un proceso relacional que se expresa a nivel de relaciones sociales generacionales entre el colectivo infantil y las personas adultas.

3. La infancia es un proceso relacional que existe, pero no únicamente en relación con la adultez y las otras generaciones coexistentes (Gaitán, 2006b: 87).

4. La sociología debiera considerar el "punto de vista de los niños", el cual apunta a entender cómo las niñas y los niños experimentan y entienden sus vidas y sus relaciones sociales (Mayall, 2002: 1).

5. La experiencia de las niñas y los niños produce un conocimiento, el cual debiera ser considerado para el reconocimiento de sus derechos (Mayall, 2002: 1).

6. El orden generacional y de género operan de modo paralelo y complementario en las jerarquías entre hombres y mujeres, y entre personas adultas y niñas y niños (Alanen, 1994: 31 y ss.). 
El enfoque relacional parte de la premisa teórica de que las niñas y los niños son actores y agentes -perspectiva desarrollada en el enfoque constructivista-, pero la acción social infantil se da dentro de parámetros de poder minoritario (los márgenes de acción minoritaria descrita en el enfoque estructural, donde se desenvuelve la vida de niñas y niños), lo que implica relaciones generacionales de poder en parte emanadas por la propia dependencia y la necesidad de protección. El hecho de que sean agentes permite considerar las visiones de ellas y ellos sobre su vida presente y no tanto las repercusiones en su futuro. Especialmente se analizan las relaciones desplegadas en espacios intrainfantiles y con las personas adultas en determinados entornos sociales como la familia, la escuela y el barrio (Mayall, 2002).

Este enfoque estudia lo que es común a las niñas y los niños en sus relaciones generacionales con las personas adultas en tanto relaciones políticas de poder entre grupos sociales a un nivel individual (micro) y a nivel grupal (macro). Por lo tanto, aquí se vuelve a recuperar la noción de la infancia como un grupo minoritario -desarrollada y presentada en el enfoque estructural- agregando la dimensión relacional de poder que está presente en la vida cotidiana. Mayall $(2002: 27,36)$ propone el término generationing (hacer generación) similar y complementario a gendering (hacer género) como un proceso mediante el cual se producen y transforman las posiciones sociales y las relaciones generacionales. Según Gaitán (2006b), el término generación se emplea para sugerir diferencias en la posición de distintos grupos de edad en virtud de su ubicación concreta en un período de la vida, a la vez que como sucesión de las actividades realizadas por los actores sociales desde una perspectiva histórica. Así surge la posibilidad sociológica de analizar los hechos desde el punto de vista de la edad y de la generación como categorías de diferenciación (Mayall, 2000, 2002: 35).

Según Mannheim (1928), la generación es una experiencia histórica que crea un marco común de vivencias e interpretaciones. El sociólogo húngaro lo estudió de modo particular para el caso de la juventud, ya que según él compartir experiencias históricas facilita una comprensión similar del mundo; así la edad se constituye en una categoría básica de diferenciación y análisis sociológico de los hechos sociales en general (Mannheim, 1928). Las premisas teóricas del estudio generacional o una perspectiva generacional que Mannheim propuso y desarrolló para el caso de la juventud Gaitán (2006b) la ha aplicado al estudio de la infancia, considerando que en cada sociedad coexisten una gran variedad de edades en cada momento presente. Por lo tanto, la perspectiva generacional permite comprender a los sujetos sociales como pertenecientes a distintas generaciones, es decir, se analizan los fenómenos a partir de un orden generacional similar al de clase o género.

\section{CONCLUSIONES}

La preocupación por la niñez desde un punto de vista sociológico, no obstante ser reciente, ha permitido una sistematización en el análisis de la infancia como componente esencial y permanente de las sociedades. Tal como se pudo apreciar, dicha preocupación ha sido recogida por los diversos enfoques que podríamos agrupar analíticamente en una propuesta teórica denominada "Sociología de la Infancia", un campo de estudios emergente 
que plantea nuevas miradas sobre la infancia como construcción social, y las niñas y los niños en tanto sujetos de derechos y actores sociales con capacidad de agencia y participación.

Los diversos enfoques de la Sociología de la Infancia rechazan el reduccionismo de separar lo individual de lo social al tomar una distancia crítica explícita de la visión que sitúa a las niñas y los niños como seres presociales y a la infancia como una etapa transitoria hacia la vida adulta. Un aporte importante de las diversas perspectivas de la Sociología de la Infancia es la comprensión de la niñez como una unidad de estudio sociológico en sí misma, aunque relacionada con la familia, la escuela, la comunidad y otros espacios sociales en que habita la niñez moderna. Desde una mirada estructural, se interpreta a la infancia como una categoría permanente en la estructura de las sociedades, aunque sus miembros se renueven constantemente. En este sentido, permite estudiar las leyes y políticas públicas destinadas al grupo infantil, considerando cómo lo afectan o cómo participan los sujetos que actualmente están en dicha posición generacional y quienes vendrán, en tanto grupo social. Por su parte, el enfoque constructivista nos entrega las herramientas conceptuales para reflexionar sobre el fenómeno de la infancia como una construcción social diversa, el cual ayuda a profundizar sobre las prácticas, valores e ideologías vigentes para la niñez en cada territorio. Por último, el enfoque relacional constituye un asidero teórico al integrar analítica y empíricamente la estructura generacional y de género en una misma matriz relacional. Los planteamientos de este último enfoque resultan vitales e innovadores para estudiar las diferentes formas y factores que inciden en la participación de las niñas y los niños en sus familias, escuelas y comunidades.

Con todo, es evidente la necesidad de repensar la infancia dentro de la sociología desde nuevos parámetros, verla como un fenómeno social y estudiar las relaciones de poder que despliegan las niñas y los niños entre sí y con las personas e instituciones adultas desde un punto de vista generacional. Tal vez esta ruptura epistemológica es similar a la ocurrida con la incorporación de la perspectiva de género en el estudio de las relaciones entre mujeres y hombres desde el poder o las relaciones étnicas en términos poscoloniales. Un paradigma sociológico adicional puede complementar y enriquecer la variedad de enfoques científicos que ya se ocupan del campo de la infancia. También en Chile es preciso avanzar hacia investigaciones que logren articular estas nuevas miradas sobre el fenómeno de la infancia, particularmente en el contexto social actual donde cada vez más las niñas, los niños y adolescentes contemporáneos presentan grandes complejidades y desafíos que son difíciles de gestionar por parte del mundo adulto.

\section{BIBLIOGRAFÍA}

Alanen, Leena (1994): "Gender and Generation: Feminism and the 'Child Question'”, en Jens Qvortrup, Marjatta Bardy, Giovanni Sgritta y Helmut Wintersberger (eds.): Childhood Matters: Social Theory, Practice and Politics, Avebury-European Centre Vienna, Vienna, Vol. 14, pp. 27-41.

Berger, Peter y Thomas Luckmann (1984 [1968]): La construcción social de la realidad, Amorrortu, Buenos Aires.

Cordero, Matías (2012): "Towards an Emancipatory Discourse of Children's Rights", International Journal of Children's Rights, 20 (3), pp. 365-421. 
Duarte Quapper, Klaudio (2001): ¿ Juventud o juventudes? Versiones, trampas, pistas y ejes para acercarnos progresivamente a los mundos juveniles, Curso "Drogas y alcohol: construir prevención desde el conocimiento integral", Universidad de Chile, Santiago.

Durkheim, Émile (1975): Educación y sociología, Península, Barcelona.

Elías, Norbert (1989): El proceso de civilización, Fondo de Cultura Económica, Madrid.

Erikson, Erik (1970): Infancia y sociedad, Hormé, Buenos Aires.

Foucault, Michel (1994 [1976]): Historia de la sexualidad, Volumen I: La voluntad de saber, Siglo XXI, Madrid.

Franzé, Adela (2002): Lo que sabía no valía. Escuela, diversidad e inmigración, Consejo Económico y Social, Comunidad de Madrid, Madrid.

Freud, Sigmund (1970): Ensayos sobre la vida sexual y la teoría de las neurosis, Alianza, Madrid.

Gaitán, Lourdes (1999a): “Bienestar social e infancia: la distribución generacional de los recursos sociales", Intervención Psicosocial, 8 (3), pp. 331-348.

(1999b): El espacio social de la infancia. Los niños en el Estado de Bienestar, Comunidad de Madrid-Conserjería de Sanidad y Servicios Sociales, Madrid.

(2006a): "La nueva sociología de la infancia. Aportaciones de una mirada distinta", Política y Sociedad, 43 (1), pp. 9-26.

(2006b): Sociología de la infancia, Síntesis, Madrid.

(dir.) (2008): Los niños como actores en los procesos migratorios, Universidad Complutense de Madrid, Madrid.

Gaitán, Lourdes y Manfred Liebel (2011): Ciudadanía y derechos de participación de los niños, Síntesis, Madrid.

Giddens, Anthony (1995 [1984]): La constitución de la sociedad: bases para la teoría de la estructuración, Amorrortu, Buenos Aires.

Gómez-Granell, Carme, Mercè Garcia-Milà, Aleix Ripol-Millet y Carme Panchón (2004): "Introducción", en Carme Gómez-Granell, Mercè Garcia-Milà y Aleix Ripol-Millet (coords.): Infancia y familias: realidades y tendencias, Ariel-CIIMU, Barcelona, pp. 15-32.

IFEJANT (2003): Infancia y adolescencia en América Latina. Aportes desde la sociología, Tomo I, IFEJANT, Lima.

James, Allison y Alan Prout (eds.) (2010 [1997]): Constructing and Reconstructing Childhood. Contemporary Issues in the Sociological Study of Childhood, Routledge, Oxon.

Jenks, Chris (ed.) (1982): The Sociology of Childhood: essential readings, Gregg Revivals, London. (2005 [1996]): Childhood, Routledge, Oxon.

Liebel, Manfred (2007): Entre protección y emancipación. Derechos de la infancia y políticas sociales, Universidad Complutense de Madrid, Madrid.

(2009): "Sobre la historia de los derechos de la infancia", en Manfred Liebel y Martínez, Marta (eds.): Infancia y derechos humanos: hacia una ciudadanía participante y protagónica, IFEJANT, Lima, pp. 23-40.

Mannheim, Karl (1993 [1928]): "El problema de las generaciones", Revista Española de Investigaciones Sociológicas, 62, pp. 193-200. 
Mayall, Berry (2000): "The Sociology of Childhood in Relation to Children's Rights", The International Journal of Children's Rights, 8, pp. 243-259.

(2009 [2002]): Towards a Sociology for Childhood. Thinking from Children's Lives, Open University Press-McGraw-Hill Education, Glasgow.

Mead, George Herbert (1982): Espíritu, persona y sociedad: desde el punto de vista del conductismo social, Paidós, Barcelona.

Moscoso, María Fernanda (2008): "Nuevos sujetos, nuevas voces: ¿hay lugar para la infancia en el pensamiento transnacional?", en Enrique Santamaría (ed.): Retos epistemológicos de las migraciones transnacionales, Anthropos, Barcelona, pp. 261-281.

Oldman, David (1994): "Adult-Child Relations as Class Relations", en Jens Qvortrup, Marjatta Bardy, Giovanni Sgritta y Helmut Wintersberger (eds.): Childhood Matters: Social Theory, Practice and Politics, Avebury-European Centre Vienna, Vienna, Vol. 14, pp. 43-58.

Parsons, Talcott. (1976 [1959]): El sistema social, Editorial Revista de Occidente, Madrid.

Pavez Soto, Iskra (2010): "Los derechos de las niñas y los niños peruanos migrantes en Chile. La infancia como un nuevo actor migratorio", Revista Enfoques, 8 (12), pp. 27-51.

(2011a): Migración infantil: rupturas generacionales y de género. Las niñas peruanas en Barcelona y Santiago de Chile, Tesis Doctoral, Departamento de Sociología, Universitat Autònoma de Barcelona, Barcelona.

(2011b): “¿Quién decide la migración infantil? Niñez y poder en familias peruanas transnacionales", RAYUELA. Revista Iberoamericana sobre Niñez y Juventud en Lucha por sus Derechos, 5, pp. 103-113.

(2012): "Inmigración y racismo: experiencias de la niñez peruana en Santiago de Chile", Si Somos Americanos. Revista de Estudios Transfronterizos, XII (1), pp. 75-99.

Piaget, Jean (1972): Psychology and Epistemology, Penguin, Harmondsworth.

(1977): The Language and Thought of the Child, Routledge \& Kegan Paul, London.

Qvortrup, Jens (1987): "Introduction to Sociology of Childhood", International Journal of Sociology, 17 (3), pp. 3-37.

(1992): "El niño como sujeto y objeto: ideas sobre el programa de infancia en el Centro europeo de Viena", Infancia y sociedad, 15, pp. 169-186.

(1994a): "Childhood Matters: An Introduction", en Jens Qvortrup, Marjatta Bardy, Giovanni Sgritta y Helmut Wintersberger (eds.): Childhood Matters: Social Theory, Practice and Politics, Avebury-European Centre Vienna, Vienna, Vol. 14, pp. 1-23.

(1994b): "Formas de acercarse a las vidas y actividades de los niños", en Investigación y políticas de infancia en Europa en los años 90, Seminario Europeo, Ministerio de Asuntos Sociales, Madrid, pp. 47-63.

Qvortrup, Jens, Marjatta Bardy, Giovanni Sgritta y Helmut Wintersberger (eds.): Childhood Matters: Social Theory, Practice and Politics, Avebury-European Centre Vienna, Vienna, Vol. 14.

Qvortrup, Jens, William A. Corsaro y Michael-Sebastian Honig (eds.) (2009): The Palgrave Handbook of Childhood Studies, Palgrave Macmillan, Basingstoke.

Rice, Philip (1997): Desarrollo humano: estudio del ciclo vital, Prentice-Hall, Madrid. 
Rodríguez, Iván (2000): “SSociología de la Infancia? Aproximaciones a un campo de estudio difuso", Revista Internacional de Sociología, 26, pp. 99-124.

Rodríguez, Iván (2007): Para una sociología de la infancia: aspectos teóricos y metodológicos, CIS, Madrid.

Rousseau Jean-Jacques (1998 [1792]): Emilio o de la educación, Alianza, Madrid.

Sánchez-Parga, José (2004): Orfandades infantiles y adolescentes. Introducción a una Sociología de la Infancia, Editorial Abya-Yala, Quito.

Unda, René (2003): "Sociología de la Infancia y Política Social ¿compatibilidades posibles?", en IFEJANT: Infancia y adolescencia en América Latina. Aportes desde la sociología, Tomo I, IFEJANT, Lima, pp. 15-29.

(2009): "Perspectivas teóricas de la sociología de la infancia en América Latina", en Manfred Liebel y Marta Martínez (eds.): Infancia y derechos humanos: hacia una ciudadanía participante y protagónica, IFEJANT, Lima, pp. 203-223.

UNICEF (1989): Convención Internacional de los Derechos del Niño, ONU, Ginebra.

Vergara, Ana, Paulina Chávez y Enrique Vergara (2010): "Televidencia y vida cotidiana de la infancia. Un estudio de casos con niños y niñas de Santiago", Polis, 9 (26), pp. 371-396.

Wartofsky, M (1981): "The Child's Construction of the World and the World's Construction of the Child: From Historical Epistemology to Historical Psychology", en F.S. Kessel y A.W. Siegel (eds.): The Child and Other Cultural Inventions, Praeger, New York, pp. 188-223.

Wasserman, Teresa (2001): “¿Quién sujeta al sujeto? Una reflexión sobre la expresión ‘el niño como sujeto de derecho'"', Ensayos y Experiencias, 8 (41), pp. 60-69.

Wintersberger, Helmut (1992): "La infancia y el cambio. Condiciones de la infancia en la Europa actual", Infancia y sociedad, 15, pp. 143-168.

(1994): "La infancia moderna", en Investigación y políticas de infancia en Europa en los años 90, Seminario Europeo, Ministerio de Asuntos Sociales, Madrid, pp. 31-46.

Recibido: 04-07-2012

Aceptado: 24-09-2012 